\title{
Improvements on the known quantum speed limits
}

Author: Harsh Anand

Affiliation: Osmania University

Correspondence: India, Telangana, Hyderabad, Secunderabad, Tarnaka, Nagarjuna Nagar, G-1 Amrutha Plaza, 12-13-673, St No. 20; Ph No. 917659912238

Email id: harshanand3.16@gmail.com

Orcid Id: 0000-0001-6903-4864

\section{Abstract:}

Let us have a Hamiltonian $\mathrm{H}$ and 2 states which can time evolve into each other via this Hamiltonian. In this particular situation, say one of the states evolves into the other in time t.

Now let us fix these two states ; then we may have infinite Hamiltonians (other than $\mathrm{H}$, say one of them is H') that can time evolve one of them into the other. But the time taken for this evolution varies from $t$ and is say t' (that is, t' is the time Hamiltonian H' takes to time evolve one of the fixed states into the other). From now on, we shall consider only those Hamiltonians that can time evolve one of the states into the other. The question is given any small positive number a, will there exist a Hamiltonian $\mathrm{H}^{\prime}$ such that t'=a ?

Answer:

Yes , the Hamiltonian Ht/ a (obtained by rescaling the original Hamiltonian) will work. But, there are bounds that show that as long as the average energy and its fluctuations are bounded (thus, no rescaling allowed), you cannot go arbitrarily fast from one state to another orthogonal state.

In this paper, we shall construct a new quantum speed limit between two arbitrary states, using only the Schrödinger equation. We shall see why mere understanding of how two fixed states may time evolve into one another in different time intervals helps construct the general quantum speed limit .

Keywords: Quantum Speed Limits, Quantum Information, Quantum computing 


\title{
Improvements on the known quantum speed limits
}

\author{
Harsh Anand, harshanand3.16@gmail.com
}

(Dated: December 2021)

\begin{abstract}
Let us have a Hamiltonian $H_{0}$ and 2 states which can time evolve into each other via this Hamiltonian. In this particular situation, say one of the states evolves into the other in time $t_{0}$.

Now let us fix these two states ; then we may have infinite Hamiltonians (other than $H_{0}$ ) that can time evolve one of them into the other. But the time taken for this evolution varies from $t_{0}$ and is say $t_{H}$ (that is, $t_{H}$ is the time Hamiltonian $\mathrm{H}$ takes to time evolve one of the fixed states into the other). From now on, we shall consider only those Hamiltonians that can time evolve one of the states into the other.

The question is given any small positive number $\epsilon$, will there exist a Hamiltonian $\mathrm{H}$ such that $t_{H}$ $=\epsilon$ ?

Answer: Yes, the Hamiltonian $H_{0} t_{0} / \epsilon$ (obtained by rescaling the original Hamiltonian) will work. But, there are bounds that show that as long as the average energy and its fluctuations are bounded (thus, no rescaling allowed), you cannot go arbitrarily fast from one state to another orthogonal state.

This discussion falls under the study of quantum speed limits. This document looks into this field of active research. We shall first give an introduction to this subject and then present a more general quantum speed limit than what is currently known.
\end{abstract}

\section{INTRODUCTION}

Quantum speed limits are rigorous estimates on how fast a state of a quantum system can depart from the initial state in the course of quantum evolution.

We first look into what work has been established so far (without going through the derivations, we state the final results, which are the known quantum speed limits and under what conditions or approximations they hold true):

We describe important milestones, the MandelstamTamm and the Margolus-Levitin bounds on the quantum speed limit.

1) The uncertainty relation of Mandelstam and Tamm: Mandelstam and Tamm proposed the first notion of a quantum speed limit :

The minimal time for a quantum system to evolve between two orthogonal states is determined by:

$$
T \geq \hbar \pi /(2 \Delta H)
$$

(For the derivation, please refer reference 3).

Uffink realised that in many situations $\Delta H$ gives not a very reasonable measure for the speed of a quantum evolution. The lower bound in the inequality can become arbitrarily small as $\Delta H$, can be infinite even if the average energy is finite.

2) The quantum speed limit of Margolus and Levitin:

To tackle this problem Margolus and Levitin proposed an alternative derivation of the quantum speed limit. They obtained the minimal evolution time between two orthogonal states as :

$$
T \geq \hbar \pi /(2<H>)
$$

Note that they explicitly assume that the average energy, $\langle H\rangle$, is non-negative.

(For the derivation, please refer reference 3)

\section{3) The unified bound is tight:}

It was finally assumed that the minimum time for time evolution between orthogonal states is given by:

$$
\mathrm{T} \geq \max (\hbar \pi /(2<H>), \hbar \pi /(2 \Delta H))
$$

However, it was Levitin and Toffoli who finally realized that the situation was not quite as simple.

They proved the following theorem:

Under the assumption that the ground state energy of a quantum systems is zero, the only state for which the Mandelstam-Tamm bound Eq. (1) as well as the Margolus-Levitin bound Eq. (2) are attained is given by

$$
\mid \Psi>=\left(\left|E_{0}>+\right| E_{1}>\right) / \sqrt{2}
$$

; where $H\left|E_{k}>=k E_{k}\right| E_{k}>$, for $\mathrm{k}=1,2$.

, and E1 is the energy of the first excited state.

II.

Now we shall present a few results that may improve the current speed limits and also may be used in wider domains.

We first note that between two fixed states, there are infinite Hamiltonians that evolve one into the other. It happens in this way: Let $\Psi$ and $\Psi^{\prime}$ be the solutions of two different Schrödinger equations. It may happen that when we plug in a value of $t=1$ say, in $\Psi$ and $t=2$ in $\Psi^{\prime}$ the expressions of both the statevectors become the same.

( For example,

$$
\Psi_{1}\left(x_{1}, t_{1}\right)=e^{-x_{1}} e^{i \sin \left(\pi t_{1} / 3\right)}+e^{-x_{1}^{2}} e^{i \cos \left(\pi t_{1} / 5\right)}
$$


and

$$
\Psi_{2}\left(x_{2}, t_{2}\right)=e^{-x_{2}} e^{i \cos \left(\pi t_{2} / 12\right)}+e^{-x_{2}^{2}} e^{i \sin \left(3 \pi t_{2} / 20\right)}
$$

These are such that they become the same expression at $t_{1}=1 \mathrm{~s}$ and $t_{2}=2 \mathrm{~s}$ respectively.)

Thus, when we are given two fixed states (they contain only the spacial coordinates), each of these states could have been formed by plugging in different values of the time coordinates present in different statevectors, which are solutions of different Schrödinger equations(formed by different Hamiltonians).

(For example,take any random Hamiltonian that forms a time dependent state vector. When values $t=t_{1}$ and $t=t_{2}$ are plugged in the statevector, two states are formed. Now consider another Hamiltonian which produces another statevector. When values $t=t_{1}^{\prime}$ and $t=t_{2}^{\prime}$ are plugged in this statevector, two states are formed.But these two states are the same states that were formed when values $t=t_{1}$ and $t=t_{2}$ were plugged in the first statevector).

Thus there are infinite Hamiltonians that evolve one fixed state to another, but the time the evolution takes varies.

Now, let us have two fixed states $\Psi\left(x, t_{1}\right)$ and $\Psi\left(x, t_{2}\right)$ such that

$$
\Psi\left(x, t_{1}\right)=\Psi^{\prime}\left(x, t_{1}^{\prime}\right), \Psi\left(x, t_{2}\right)=\Psi^{\prime}\left(x, t_{2}^{\prime}\right)
$$

where $\Psi^{\prime}$ is a sample statevector (it is such that it passes through the two fixed states). We note that

$\left\|\Psi\left(x, t_{1}\right)-\Psi\left(x, t_{2}\right)\right\|$ is a constant (as the Hamiltonians are varied).

Let us consider one particular path (one particular Hamiltonian) from one of the states to the other. We shall prove this inequality :

$$
\left\|\Psi\left(x, t_{1}\right)-\Psi\left(x, t_{2}\right)\right\| \leq \int_{t_{1}}^{t_{2}}{\sqrt{<H^{2}}>_{\Psi(x, t)}} d t / \hbar
$$

in two different ways

1)

Proof:

We have

$$
\mathrm{i} \hbar \partial \Psi / \partial t=H \Psi
$$

That is,

$$
\Psi(t+d t)-\Psi(t)=d t H \Psi(t) /(i \hbar)
$$

Taking norm on both sides, we get

$$
\|\Psi(t+d t)-\Psi(t)\| \hbar=\sqrt{<H^{2}>_{\Psi}(x, t)} d t
$$

Then, for times $t_{1}$ and $t_{2}$

$\left\|\Psi\left(t_{2}\right)-\Psi\left(t_{1}\right)\right\| \leq\left\|\Psi\left(t_{2}\right)-\Psi\left(t_{2}-d t\right)\right\|+\ldots .|| \Psi\left(t_{1}+\right.$ $d t)-\Psi\left(t_{1}\right) \|$

Thus,

$=\Sigma{\sqrt{<H^{2}>_{\Psi}(x, t)}} d t / \hbar=\int_{t_{1}}^{t_{2}} \sqrt{<H^{2}>_{\Psi}(x, t)} d t / \hbar$

$$
\left\|\Psi\left(t_{2}\right)-\Psi\left(t_{1}\right)\right\| \leq \int_{t_{1}}^{t_{2}} \sqrt{<H^{2}>_{\Psi}(x, t)} d t / \hbar
$$

Hence proved.

2) We have

$$
\mathrm{i} \hbar \partial \Psi / \partial t=H \Psi
$$

By the taylor expansion, we have, $f(t+d t)=f(t)+$ $f^{\prime}(t) d t+\ldots$ Here we have $f(t)=\Psi(t)$ and $f^{\prime}(t)=$ $H \Psi(t) /(i \hbar)$. Thus, on expanding

$$
\Psi(t+d t)=\Psi(t)+d t H \Psi(t) /(i \hbar)-(d t)^{2} H^{2} \Psi(t) / \hbar^{2}+\ldots
$$

Applying the bra vector $<\Psi(t) \mid$ on both sides of eq (12) and then taking it's real part into consideration, we have:

$$
\operatorname{Re}(<\Psi(t) \mid \Psi(t+d t)>)=1-(\mathrm{dt})^{2}<H^{2}>_{\Psi(t)} / \hbar^{2}
$$

Now, for any times $t_{1}$ and $t_{2}$, if $\mathrm{U}$ is the unitary time evolver of the system,

$$
\left\|\Psi\left(t_{1}\right)-\Psi\left(t_{2}\right)\right\|^{2}=\left\|U\left(t_{2}, t_{1}\right) \Psi\left(t_{1}\right)-\Psi\left(t_{1}\right)\right\|^{2}
$$

$=$ after much simplification,

$$
2-2 \operatorname{Re}\left(<\Psi\left(t_{2}\right) \mid \Psi\left(t_{1}\right)>\right)
$$

In $\mathrm{Eq}(15)$, substituting $t_{2}$ for $\mathrm{t}+\mathrm{dt}, t_{1}$ for $\mathrm{t}$, we combine Eqs.(13) and (15)

$$
\|\Psi(t+d t)-\Psi(t)\| \hbar={\sqrt{<H^{2}>_{\Psi}(x, t)}} d t
$$

Then, for times $t_{1}$ and $t_{2}$

$\left\|\Psi\left(t_{2}\right)-\Psi\left(t_{1}\right)\right\| \leq\left\|\Psi\left(t_{2}\right)-\Psi\left(t_{2}-d t\right)\right\|+\ldots .|| \Psi\left(t_{1}+\right.$ $d t)-\Psi\left(t_{1}\right) \|$

$=\Sigma{\sqrt{\left.<H^{2}\right\rangle_{\Psi(x, t)}}}_{t} / \hbar=\int_{t_{1}}^{t_{2}}{\sqrt{\left.<H^{2}\right\rangle_{\Psi(x, t)}}}_{t / \hbar}$ Thus,

$$
\left\|\Psi\left(t_{2}\right)-\Psi\left(t_{1}\right)\right\| \leq \int_{t_{1}}^{t_{2}} \sqrt{<H^{2}>_{\Psi}(x, t)} d t / \hbar
$$

Hence proved.

\section{INFERENCES}

1)

$$
\left\|\Psi\left(x, t_{1}\right)-\Psi\left(x, t_{2}\right)\right\| \leq \int_{t_{1}}^{t_{2}}{\sqrt{<H^{2}}>_{\Psi(x, t)}} d t / \hbar
$$

Now this inequality is an inequality satisfied by each path the initial state takes to evolve into the final state. The interesting thing to observe is that by $\mathrm{Eq}(5)$,

$\Psi\left(x, t_{1}\right)=\Psi^{\prime}\left(x, t_{1}^{\prime}\right), \Psi\left(x, t_{2}\right)=\Psi^{\prime}\left(x, t_{2}^{\prime}\right)$ where the primes denote any other system which passes through the two fixed states in it's time evolution by the system's Hamiltonian. In this other system, the inequality is 


$$
\left\|\Psi^{\prime}\left(x, t_{1}^{\prime}\right)-\Psi^{\prime}\left(x, t_{2}^{\prime}\right)\right\| \leq \int_{t_{1}^{\prime}}^{t_{2}^{\prime}} \sqrt{<H^{2}>_{\Psi^{\prime}}}{ }_{\left(x, t^{\prime}\right)} d t^{\prime} / \hbar
$$

That is, the other system's statevector is $\Psi^{\prime}$, it passes through the two fixed states in times $t_{1}^{\prime}$ and $t_{2}^{\prime}$ respectively.

Here,

$$
\left\|\Psi\left(x, t_{1}\right)-\Psi\left(x, t_{2}\right)\right\|=\left\|\Psi^{\prime}\left(x, t_{1}^{\prime}\right)-\Psi^{\prime}\left(x, t_{2}^{\prime}\right)\right\|
$$

That means, the right hand side of the inequality, the larger term, is always greater than a fixed number, as the Hamiltonians (and thus the right hand side of the inequality, the integrals) are varied. So if we choose a Hamiltonian such that it time evolves one of the states into the other in a short time $d t^{\prime}$, the integral will approximately become

$$
\sqrt{<H^{2}>_{\Psi}}{ }_{\left(x, t^{\prime}\right)} d t^{\prime} / \hbar
$$

Even with the term $d t^{\prime}$ multiplied, this is larger than the constant. That means

$$
{\sqrt{<H^{2}>_{\Psi}}}_{\left(x, t^{\prime}\right) / \hbar}
$$

has to be sufficiently large. The energy of the system, to some accuracy is given by this term, and thus, if we need the system to time evolve from one state to the other in a short time interval, we need the energy to be given to the system as sufficiently large.

$$
\int_{t_{1}}^{t_{2}} \sqrt{<H^{2}>_{\Psi}(x, t)} d t /\left(t_{2}-t_{1}\right)
$$

may be defined as the time average of the energies in the evolving states. Thus it is sort of an average of many averages.

\section{3)}

The inequality when applied to dynamics of two state systems or the most general time dependent three state systems always seems to reduce to the inequality $|\sin (x)| \leq|x|$. For more details on the dynamics of quantum three state systems, please refer reference 2 .

4)

In the introduction, we had seen that the known quantum speed limits had certain drawbacks. Namely:

a) They were useful only when considering the the evolution between two orthogonal states.

b) They were correct only for states of the form given by $\operatorname{Eq}(4)$.

Now the inequality that has been derived overcomes these drawbacks and gives an accurate quantum speed limit between any two states( which need not be orthogonal, nor be of the form of $\mathrm{Eq}(4))$.

The problem of finding the speed limit for transition from one state to another presents by it's very construction the idea of varying the Hamiltonians between two fixed states. When we vary the Hamiltonians, the time values of the time at which the states have been evolved into or from, change. We have seen this. Thus to find the minimum time a state would take to get transformed into another state, we may consider only those Hamiltonians that do this evolution in a very short time.

Then it is clear that the new quantum speed limit of transition from one fixed state to another is the liminf of:

$$
\left\|\Psi\left(x, t_{1}\right)-\Psi\left(x, t_{2}\right)\right\| \hbar /{\sqrt{<H^{2}>_{\Psi}}}^{\prime}\left(x, t^{\prime}\right)
$$

Where liminf is the infimum value of all the numbers given in $\mathrm{Eq}(18)$. $\mathrm{Eq}(18)$ has numbers formed by Hamiltonians that do the time evolution of the two states very quickly. Each time the process of time evolution of the two states is done by a Hamiltonian $\mathrm{H}$ (quickly), the time for evolution by this particular $\mathrm{H}$ is greater than the expresssion in $\mathrm{Eq}(18)$. Thus the infimum value of all the numbers in $\mathrm{Eq}(18)$ would give the required minimum time for time evolution of one of the fixed states into the other. Note that this minimum time can be close to zero only if

$$
\sqrt{<H^{2}>_{\Psi}}{ }_{\left(x, t^{\prime}\right)}
$$

is very large for some $\mathrm{H}$, where $\mathrm{H}$ is one of the Hamiltonians that does the time evolution.

\section{ACKNOWLEDGMENTS}

The author would like to thank the excellent courses on edX on Quantum Mechanics by MIT ;the illustrious professor Barton Zwiebach's inspiring persona which instills a sense of curiosity along with ambition in the students; the ever helpful Mr. Mark Weitzman ; and Mr. Alex Shvonski, with whose support and motivation, this paper has been developed.

\section{REFERENCES}

1) https://arxiv.org/pdf/quant-ph/9710043.pdf

The maximum speed of dynamical evolution

2) Arbitrary quantum state engineering in three-state systems via Counterdiabatic driving. :

Ye-Hong Chen, Qi-Cheng Wu; Scientific Reports Article number: 38484

3) Quantum speed limits: from Heisenberg's uncertainty principle to optimal quantum control:

https://arxiv.org/pdf/1705.08023.pdf

4)

a)

8.05 Mastering Quantum mechanics, Barton Zwiebach, MIT.

b)

8.04 Quantum Mechanics: A First Course

5) https://physics.stackexchange.com/questions/680988/varyingthe-hamiltonians-between-two-fixedstates/681037681037

6) H. J. Bremermann. Quantum noise and information. In Proceedings of the Fifth Berkeley Symposium 
on Mathematical Statistics and Probability, Volume 4: Biology and Problems of Health, pages 15-20, Berkeley, Calif., 1967. University of California Press.

7) J. D. Bekenstein. Energy Cost of Information Trans- fer. Phys. Rev. Lett., 46:623, 1981.

8) J. D. Bekenstein. Generalized second law of thermodynamics in black-hole physics. Phys. Rev. D, 9:3292, 1974. 